\title{
Upper bounds for number of removed edges in the Erased Configuration Model
}

\author{
Pim van der Hoorn ${ }^{1}$ and Nelly Litvak ${ }^{1}$ \\ University of Twente, Department of Electrical Engineering, Mathematics and \\ Computer Science \\ w.l.f.vanderhoorn@utwente.nl
}

\begin{abstract}
Models for generating simple graphs are important in the study of real-world complex networks. A well established example of such a model is the erased configuration model, where each node receives a number of half-edges that are connected to half-edges of other nodes at random, and then self-loops are removed and multiple edges are concatenated to make the graph simple. Although asymptotic results for many properties of this model, such as the limiting degree distribution, are known, the exact speed of convergence in terms of the graph sizes remains an open question. We provide a first answer by analyzing the size dependence of the average number of removed edges in the erased configuration model. By combining known upper bounds with a Tauberian Theorem we obtain upper bounds for the number of removed edges, in terms of the size of the graph. Remarkably, when the degree distribution follows a power-law, we observe three scaling regimes, depending on the power law exponent. Our results provide a strong theoretical basis for evaluating finite-size effects in networks.
\end{abstract}

\section{Introduction}

The use of complex networks to model large systems has proven to be a powerful tool in recent years. Mathematical and empirical analysis of structural properties of such networks, such as graph distances, centralities, and degree-degree correlations, have received vast attention in recent literature. A common approach for understanding these properties on real-world networks, is to compare them to those of other networks which have the same basic characteristics as the network under consideration, for instance the distribution of the degrees. Such test networks are usually created using random graph models. An important property of real-world networks is that they are usually simple, i.e. there is at most one edge between any two nodes and there are no self-loops. Hence, random graph models that produce simple graphs are of primary interest from the application point of view.

One well established model for generating a graph with given degree distribution is the configuration model [5, 19, 21, which has been studied extensively in the literature [6, 12, 15, 16. In this model, each node first receives a certain number of half-edges, or stubs, and then the stubs are connected to each other 
at random. Obviously, multiple edges and self-loops may appear during the random wiring process. It is well-known that when the degree distribution has finite variance, the graph will be simple with positive probability, so a simple graph can be obtained by repeatedly applying the model until the resulting graph is simple. However, when the variance of the degrees is infinite the resulting graph will, with high probability, not be simple. To remedy this, one can remove selfloops and concatenate the multiple edges to make the graph simple. This version is know as the erased configuration model. Although removal of edges impacts the degree distribution, it has been shown that asymptotically the degree distribution is unchanged. For a thorough systematic treatment of these results we refer the reader to 12 .

An important feature of the configuration model is that, conditioned on the graph being simple, it samples a graph uniformly from among all simple graphs with the specified degree distribution. This, in combination with the neutral wiring in the configuration model, makes it a crucial model for studying the effects of degree distributions on the structural properties of the networks, such as, for instance, graph distances [10, 13, 14, 20] and epidemic spread 11, 11, 17.

We note that there are several different methods for generating simple graphs, sampled uniformly from the set of all simple graphs with a given degree sequence. A large class of such models use Markov-Chain Monte Carlo methods for sampling graphs uniformly from among all graphs with a given set of constraints, such as the degree sequence. These algorithms use so-called edge swap or switching steps, 2, 18, 23, each time a pair of edges is sampled and swapped, if this is allowed. The main problem with this method are the limited theoretical results on the mixing times, in [7 mixing times are analyzed, but only for regular graphs. Other methods are, for instance, the sequential algorithms proposed in [4, 8] which have complexity $O\left(E N^{2}\right)$ and $O(E N)$, respectively, where $N$ is the size of the graph and $E$ denotes the number of edges. The erased configuration model however,is well studied, with strong theoretical results and is easy to implement.

In a recent study [22], authors compare several methods, including the above mentioned Markov-Chain Monte Carlo methods, for creating test graphs for the analysis of structural properties of networks. The authors found that the number of removed edges did not impact the degree sequence in any significant way. However, several other measures on the graph, for instance average neighbor degree, did seem to be altered by the removal of self-loops and double-edges. This emphasizes the fact that asymptotic results alone are not sufficient. The analysis of networks requires a more detailed understanding of finite-size effects in simple random graphs. In particular, it is important to obtain a more precise characterization for dependence of the number of erased edges on the graph size, and their impact on other characteristics of the graph.

In our recent work [16] we analyzed the average number of removed edges in order to evaluate the degree-degree correlations in the directed version of the erased configuration model. We used insights obtained from several limit theorems to derive the scaling in terms of the graph size. Here we make these 
rigorous by proving three upper bounds for the average number of removed edges in the undirected erased configuration model with regularly varying degree distribution. We start in Section 2 with the formal description of the model. Our main result is stated in Section 3 and the proofs are provided in Section 4.

\section{Erased Configuration Model}

The Erased Configuration Model (ECM) is an alteration of the Configuration Model (CM), which is a random graph model for generating graphs of size $n$ with either prescribed degree sequence or degree distribution. Given a degree sequence $\mathbf{D}_{n}$ such that $\sum_{i=1}^{n} D_{i}$ is even, the degrees of each node are represented as stubs and the graph is constructed by randomly pairing stubs to form edges. This will create a graph with the given degree sequence.

In another version of the model, degrees are sampled independently from a given distribution, an additional stub is added to the last node if the sum of degrees is odd, and the stubs are connected as in the case with given degrees. The empirical degree distribution of the resulting graph will then converge to the distribution from which the degrees were sampled as the graph size goes to infinity, see for instance [12.

When the degree distribution has finite variance, the probability of creating a simple graph with the CM is bounded away from zero. Hence, by repeating the model, one will obtain a simple graph after a finite number of attempts. This construction is called the Repeated Configuration Model (RCM). It has been shown that the RCM samples graphs uniformly from among all simple graphs with the given degree distribution, see Proposition 7.13 in [12].

When the degrees have infinite variance the probability of generating a simple graph with the CM converges to zero as the graph size increases. In this case the ECM can be used, where after all stubs are paired, multiple edges are merged and self-loops are removed. This model is computationally far less expensive than the RCM since the pairing only needs to be done once while in the other case the number of attempts increases as the variance of the degree distribution grows. The trade-off is that the ECM removes edges, altering the degree sequence and hence the empirical degree distribution. Nevertheless it was proven, see [12, that the empirical degree distribution for the ECM still converges to the original one as $n \rightarrow \infty$.

For our analysis we shall consider graphs of size $n$ generated by the ECM, where the degrees are sampled at random from a regularly varying distribution. We recall that $X$ is said to have a regularly varying distribution with finite mean if

$$
\mathbb{P}(X>k)=L(k) k^{-\gamma} \quad \text { with } \gamma>1,
$$

where $L$ is a slowly varying function, i.e. $\lim _{x \rightarrow \infty} L(t x) / L(x)=1$ for all $t>0$. The parameter $\gamma$ is called the exponent of the distribution.

For $n \in \mathbb{N}$ we consider the degree sequence $\mathbf{D}_{n}$ as a sequence of i.i.d. samples from distribution (1), let $\mu=\mathbb{E}[D]$ and denote by $L_{n}=\sum_{i=1}^{n} D_{i}$ the sum of the degrees. Formally we need $L_{n}$ to be even in order to have a graphical sequence, 
in which case $L_{n} / 2$ is the number of edges. This can be achieved by increasing the degree of the last node $D_{n}$ by one if the sum is odd. This alteration adds a term uniformly bounded by one which does not influence the analysis. Therefore we can omit this and treat the degree sequence $\mathbf{D}_{n}$ as an i.i.d. sequence.

For the analysis we denote by $E_{i j}$ the number of edges between two nodes, $1 \leq i, j \leq n$, created by the CM and by $E_{i j}^{c}$ the number of edges between the two nodes that where removed by the ECM. Furthermore, we let $\mathbb{P}_{n}$ and $\mathbb{E}_{n}$ be, respectively, the probability and expectation conditioned on the degree sequence $\mathbf{D}_{n}$.

\section{Main result}

The main result of this paper is concerned with the scaling of the average number of erased edges in the ECM. It was proven in [15] that

$$
\frac{1}{L_{n}} \sum_{i, j} \mathbb{E}_{n}\left[E_{i j}^{c}\right] \stackrel{\mathbb{P}}{\rightarrow} 0 \quad \text { as } n \rightarrow \infty
$$

where $\stackrel{\mathbb{P}}{\rightarrow}$ denotes convergence in probability. This result states that the average number of removed edges converges to zero as the graph size grows, which is in agreement with the convergence in probability of the empirical degree distribution to the original one. However, until now there have not been any results on the speed of convergence in (2). In this section we will state our result, which establishes upper bound on the scaling of the average number of erased edges.

To make our statement rigorous we first need to define what we mean by scaling for a random variable.

Definition 1. Let $\left(X_{n}\right)_{n \in \mathbb{N}}$ be sequences of random variables and let $\rho \in \mathbb{R}$. Then we define

$$
X_{n}=O_{\mathbb{P}}\left(n^{\rho}\right) \Longleftrightarrow \text { for all } \varepsilon>0 \quad n^{-\rho-\varepsilon} X_{n} \stackrel{\mathbb{P}}{\rightarrow} 0, \quad \text { as } n \rightarrow \infty .
$$

We are now ready to state the main result on the scaling of the average number of erased edges in the ECM

Theorem 1. Let $G_{n}$ be a graph generated by the ECM with degree distribution (11), let $L_{n}$ be the sum of the degrees and denote by $E_{i j}^{c}$ the number of removed edges from $i$ to $j$. Then

$$
\frac{1}{L_{n}} \sum_{i, j=1}^{n} \mathbb{E}_{n}\left[E_{i j}^{c}\right]= \begin{cases}O_{\mathbb{P}}\left(n^{\frac{1}{\gamma}-1}\right) & \text { if } 1<\gamma \leq \frac{3}{2}, \\ O_{\mathbb{P}}\left(n^{\frac{4}{\gamma}-3}\right) & \text { if } \frac{3}{2}<\gamma \leq 2, \\ O_{\mathbb{P}}\left(n^{-1}\right) & \text { if } \gamma>2 .\end{cases}
$$

The proof of Theorem 1 is given in the next section. The strategy of the proof is to establish two upper bounds for $\sum_{i, j=1}^{n} \mathbb{E}_{n}\left[E_{i j}^{c}\right] / L_{n}$ for the case $1<\gamma \leq 2$, 
each of which scales as one of the first two terms from (3). Then it remains to observe that the term $n^{1 / \gamma-1}$ dominates $n^{4 / \gamma-3}$ when $1<\gamma \leq 3 / 2$ while the latter one dominates when $3 / 2<\gamma<2$. In addition, we prove the $n^{-1}$ scaling for $\gamma>2$.

Theorem 1 gives several insights into the behavior of the ECM. First, consider the case when the degrees have finite variance $(\gamma>2)$. Equation (3) tells us that in that case the ECM will erase only a finite, in $n$, number of edges. For large $n$, this alters the degree sequence in a negligible way. We then gain the advantage that we need to perform the random wiring only once. In contrast, the RCM requires multiple attempts before a simple graph is produced. This will be a problem, especially as $\gamma$ approaches 2 .

An even more interesting phenomenon established by Theorem 1 is the remarkable change in the scaling at $\gamma=3 / 2$. Figure 1 shows the exponent in the scaling term in (3) as a function of $\gamma$. Notice that for small values of $\gamma$, the frac-

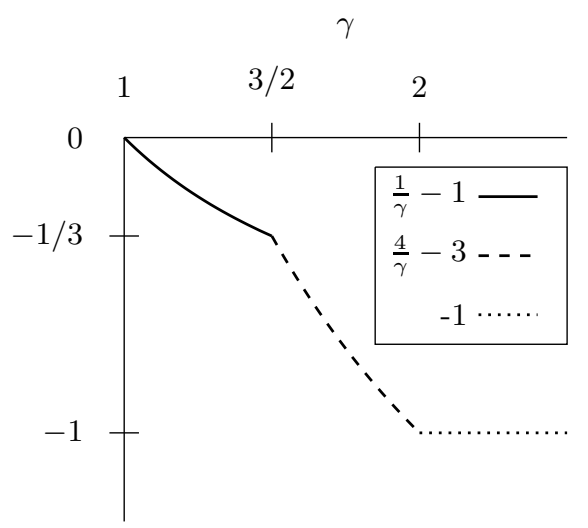

Fig. 1. The scaling exponent of the average number of erased edges, as a function of $\gamma$.

tion of erased edges decreases quite slowly with $n$. For example, when $\gamma=1.1$ and $n=10^{6}$ then $n^{1 / \gamma} \approx 284803$. Hence, a significant fraction of edges will be removed, so we can expect notable finite size effects even in large networks. However, when $\gamma \geq 1.5$ the finite size effects are already very small and decrease more rapidly with $\gamma$.

It will be seen from the proofs in the next section that the upper bounds for $\gamma>3 / 2$ in Theorem 1 follow readily from the literature. Our main contribution is in the upper bound for $1<\gamma<3 / 2$, which corresponds to many real-world networks. The proof uses a Central Limit Theorem and a Tauberian Theorem for regularly varying random variables. Note that when $1<\gamma<3 / 2$ the upper bound $n^{4 / \gamma-3}$ is not at all tight and even increasing in $n$ for $\gamma<4 / 3$. 


\section{Upper bounds for erased edges}

Throughout this section we will use the Central Limit Theorem for regularly varying random variables also called the Stable Law CLT, see 24 Theorem 4.5.1. We summarize it below, letting $\stackrel{d}{\rightarrow}$ denote convergence in distribution, in the setting of non-negative regularly varying random variables.

Theorem 2 (Stable Law CLT [24]). Let $\left\{X_{i}: i \geq 1\right\}$ be an i.i.d. sequence of non-negative random variables with distribution (11) and $0<\gamma<2$. Then there exists a slowly varying function $L_{0}$, different from $L$, such that

$$
\frac{\sum_{i=1}^{n} D_{i}-m_{n}}{L_{0}(n) n^{\frac{1}{\gamma}}} \stackrel{d}{\rightarrow} \mathcal{S}_{\gamma}
$$

where $S_{\gamma}$ is a stable random variable and

$$
m_{n}= \begin{cases}0 & \text { if } 0<\gamma<1 \\ n^{2} \mathbb{E}\left[\sin \left(\frac{X}{L_{0}(n) n}\right)\right] & \text { if } \gamma=1 \\ n \mathbb{E}[X] & \text { if } 1<\gamma<2 .\end{cases}
$$

From Theorem 2 we can infer several scaling results using the following observation: By Slutsky's Theorem it follows that

$$
\frac{\sum_{i=1}^{n} X_{i}-m_{n}}{L_{0}(n) n^{\frac{1}{\gamma}}} \stackrel{d}{\rightarrow} S_{\gamma} \quad \text { as } n \rightarrow \infty
$$

implies that for any $\varepsilon>0$,

$$
n^{-\varepsilon} \frac{\sum_{i=1}^{n} X_{i}-m_{n}}{L_{0}(n) n^{\frac{1}{\gamma}}} \stackrel{\mathbb{P}}{\rightarrow} 0 \quad \text { as } n \rightarrow \infty .
$$

Hence $\left|\sum_{i=1}^{n} X_{i}-m_{n}\right|=O_{\mathbb{P}}\left(L_{0}(n) n^{1 / \gamma}\right)$ and therefore, by Potter's Theorem, it follows that $\left|\sum_{i=1}^{n} X_{i}-m_{n}\right|=O_{\mathbb{P}}\left(n^{1 / \gamma}\right)$. Finally, we remark that if $D$ has distribution (11) with $1<\gamma \leq 2$, then $D^{2}$ has distribution (1) with exponent $1 / 2<\gamma / 2 \leq 1$. Summarizing, we have the following.

Corollary 1. Let $G_{n}$ be a graph generated by the ECM with degree distribution (11) and $1<\gamma \leq 2$, then

$$
L_{n}=O_{\mathbb{P}}(n), \quad\left|\sum_{i=1}^{n} D_{i}-\mu n\right|=O_{\mathbb{P}}\left(n^{\frac{1}{\gamma}}\right) \text { and } \sum_{i=1}^{n} D_{i}^{2}=O_{\mathbb{P}}\left(n^{\frac{2}{\gamma}}\right)
$$

The third equation also holds for $\gamma=2$ since

$$
\begin{aligned}
\sum_{i=1}^{n} D_{i}^{2} & =\left(\sum_{i=1}^{n} D_{i}^{2}-L_{0}(n) n^{2} \mathbb{E}\left[\sin \left(\frac{D}{n L_{0}(n)}\right)\right]\right)+L_{0}(n) n^{2} \mathbb{E}\left[\sin \left(\frac{D}{n^{1} L_{0}(n)}\right)\right] \\
& \leq\left(\sum_{i=1}^{n} D_{i}^{2}-n^{2} L_{0}(n) \mathbb{E}\left[\sin \left(\frac{D}{n^{1} L_{0}(n)}\right)\right]\right)+n \mu \\
& =O_{\mathbb{P}}\left(L_{0}(n) n\right)+n \mu=O_{\mathbb{P}}(n) .
\end{aligned}
$$




\subsection{The upper bounds $O_{\mathbb{P}}\left(n^{\frac{4}{\gamma}-3}\right)$ and $O_{\mathbb{P}}\left(n^{-1}\right)$}

For the proof of the upper bounds we will use the following proposition.

Proposition 1 (Proposition 7.10 [12]). Let $G_{n}$ be a graph generated by the $C M$ and denote by $S_{n}$ and $M_{n}$, respectively, the number of self-loops and multiple edges. Then

$$
\mathbb{E}_{n}\left[S_{n}\right] \leq \sum_{i=1}^{n} \frac{D_{i}^{2}}{L_{n}} \quad \text { and } \quad \mathbb{E}_{n}\left[M_{n}\right] \leq 2\left(\sum_{i=1}^{n} \frac{D_{i}^{2}}{L_{n}}\right)^{2} .
$$

Lemma 1. Let $G_{n}$ be a graph generated by the ECM with degree distribution (11), then

$$
\frac{1}{L_{n}} \sum_{i, j=1}^{n} \mathbb{E}_{n}\left[E_{i j}^{c}\right]= \begin{cases}O_{\mathbb{P}}\left(n^{\frac{4}{\gamma}-3}\right) & \text { if } 1<\gamma \leq 2, \\ O_{\mathbb{P}}\left(n^{-1}\right) & \text { if } \gamma>2 .\end{cases}
$$

Proof. We start by observing that

$$
\sum_{i, j=1}^{n} E_{i j}^{c}=S_{n}+M_{n}
$$

and hence it follows from Proposition 1 that

$$
\sum_{i, j=1}^{n} \mathbb{E}_{n}\left[E_{i j}^{c}\right] \leq \sum_{i=1}^{n} \frac{D_{i}^{2}}{L_{n}}+2\left(\sum_{i=1}^{n} \frac{D_{i}^{2}}{L_{n}}\right)^{2}
$$

First suppose that $1<\gamma \leq 2$. Then, by Corollary 1 and the continuous mapping theorem it follows that

$$
\frac{1}{L_{n}} \sum_{i, j=1}^{n} \mathbb{E}_{n}\left[E_{i j}^{c}\right] \leq \frac{1}{L_{n}^{2}} \sum_{i=1}^{n} D_{i}^{2}+2 \frac{1}{L_{n}^{3}}\left(\sum_{i=1}^{n} D_{i}^{2}\right)^{2}=O_{\mathbb{P}}\left(n^{\frac{4}{\gamma}-3}\right) .
$$

Now suppose that $\gamma>2$. Then $D_{i}^{2}$ has finite mean, say $\nu$, and therefore, by Theorem 2,

$$
\frac{1}{L_{n}^{2}} \sum_{i=1}^{n} D_{i}^{2} \leq \frac{1}{L_{n}^{2}}\left|\sum_{i=1}^{n} D_{i}^{2}-n \nu\right|+\frac{n \nu}{L_{n}^{2}}=O_{\mathbb{P}}\left(n^{\frac{2}{\gamma}-2}+n^{-1}\right)=O_{\mathbb{P}}\left(n^{-1}\right),
$$

where the last step follows since $2 / \gamma-2<-1$ when $\gamma>2$. Since this is the main term the result follows.

Lemma 1 provides the last two upper bounds from Theorem 1. However, as we mentioned before, the bound $O_{\mathbb{P}}\left(n^{4 / \gamma-3}\right)$ is not tight over the whole range $1<\gamma \leq 2$ since for $1<\gamma<4 / 3$ we have $4 / \gamma-3>0$, and hence the upper bound diverges as $n \rightarrow \infty$ which is in disagreement with (2). Therefore, there must exist another upper bound on the average erased number of edges, which goes to zero as $n \rightarrow \infty$ for all $\gamma>1$. This new bound does not follow readily from the literature. Below we establish such upper bound and explain the essential new ingredients needed for its proof. 


\subsection{The upper bound $O_{\mathbb{P}}\left(n^{\frac{1}{\gamma}-1}\right)$}

We first observe that the number of erased edges between nodes $i$ and $j$ equals the total number of edges between the nodes minus one, if there is more than one edge. This gives,

$$
\begin{aligned}
\frac{1}{L_{n}} \sum_{i, j=1}^{n} \mathbb{E}_{n}\left[E_{i j}^{c}\right] & =\frac{1}{L_{n}} \sum_{i, j=1}^{n} \mathbb{E}_{n}\left[E_{i j}-\mathbb{1}_{\left\{E_{i j}>0\right\}}\right] \\
& =\frac{1}{L_{n}} \sum_{i, j=1}^{n} \mathbb{E}_{n}\left[E_{i j}\right]-\frac{1}{L_{n}} \sum_{i, j=1}^{n} \mathbb{E}_{n}\left[1-\mathbb{1}_{\left\{E_{i j}=0\right\}}\right] \\
& =1-\frac{n^{2}}{L_{n}}+\frac{1}{L_{n}} \sum_{i, j=1}^{n} \mathbb{P}_{n}\left(E_{i j}=0\right)
\end{aligned}
$$

We can get an upper bound for $\mathbb{P}_{n}\left(E_{i j}=0\right)$ from the analysis performed in [13, Section 4. Since the probability of no edges between $i$ and $j$ equals the probability that none of the $D_{i}$ stubs connects to one of the $D_{j}$ stubs, it follows from equation (4.9) in [13] that

$$
\mathbb{P}_{n}\left(E_{i j}=0\right) \leq \prod_{k=0}^{D_{i}-1}\left(1-\frac{D_{j}}{L_{n}-2 D_{i}-1}\right)+\frac{D_{i}^{2} D_{j}}{\left(L_{n}-2 D_{i}\right)^{2}}
$$

The product term in (6) can be upper bounded by $\exp \left\{-D_{i} D_{j} / E_{n}\right\}$. For the second term we use that

$$
\begin{aligned}
\frac{1}{L_{n}} \sum_{i, j=1}^{n} \frac{D_{i}^{2} D_{j}}{\left(L_{n}-2 D_{i}\right)^{2}} & =\frac{1}{L_{n}^{2}} \sum_{i=1}^{n} D_{i}^{2}\left(\frac{1}{1-\frac{2 D_{i}}{L_{n}}}\right)^{2}\left(\frac{1}{L_{n}} \sum_{j=1}^{n} D_{j}\right) \\
& \leq \frac{1}{L_{n}^{2}} \sum_{i=1}^{n} D_{i}^{2}=O_{\mathbb{P}}\left(n^{\frac{2}{\gamma}-2}\right) .
\end{aligned}
$$

Putting everything together we obtain

$$
\frac{1}{L_{n}} \sum_{i, j=1}^{n} \mathbb{P}_{n}\left(E_{i j}=0\right) \leq \sum_{i, j=1}^{n} \exp \left\{-\frac{D_{i}^{+} D_{j}^{-}}{L_{n}}\right\}+O_{\mathbb{P}}\left(n^{\frac{2}{\gamma}-2}\right) .
$$

We will use (7) to upper bound (5). In order to obtain the desired result we will employ a Tauberian Theorem for regularly varying random variables, which we summarize first. We write $a \sim b$ to denote that $a / b$ goes to one in a corresponding limit. 
Theorem 3 (Tauberian Theorem, [3]). Let $X$ be a non-negative random variable with only finite mean. Then, for $1<\gamma<2$, the following are equivalent,

i) $\mathbb{P}(X>t) \sim L(t) t^{-\gamma}$ as $t \rightarrow \infty$,

ii) $\frac{\mathbb{E}[X]}{t}-1+\exp \left\{-\frac{X}{t}\right\} \sim L\left(\frac{1}{t}\right) t^{-\gamma}$ as $t \rightarrow \infty$.

We will first explain the idea behind the proof of the $O_{\mathbb{P}}\left(n^{1 / \gamma-1}\right)$ bound. If we insert (7) into (5) we get

$$
\frac{1}{L_{n}} \sum_{i, j=1}^{n} \mathbb{E}_{n}\left[E_{i j}^{c}\right] \leq 1-\frac{n^{2}}{L_{n}}+\frac{1}{L_{n}} \sum_{i, j=1}^{n} \exp \left\{-\frac{D_{i}^{+} D_{j}^{-}}{L_{n}}\right\}+O_{\mathbb{P}}\left(n^{\frac{2}{\gamma}-2}\right) .
$$

The terms on the right side can be rewritten to obtian an expression that resembles an empirical version of the left hand side of part ii) from Theorem 3, with $t=L_{n}$ and $X=D_{1} D_{2}$. Thus, the scaling of the average number of erased edges will be determined by the scaling that follows from the Tauberian Theorem and the Stable Law CLT.

Proposition 2. Let $G_{n}$ be a graph generated by the ECM with degree distribution (11) and $1<\gamma<2$. Then

$$
\frac{1}{L_{n}} \sum_{i, j=1}^{n} \mathbb{E}_{n}\left[E_{i j}^{c}\right]=O_{\mathbb{P}}\left(n^{\frac{1}{\gamma}-1}\right)
$$

Proof. We start with equation (8). Since the correction term here is of lower order, by extracting a factor $n^{2} / L_{n}$ from the other terms and using that $L_{n}=$ $\sum_{i=1}^{n} D_{i}$, it suffices to show that

$$
\frac{n^{2}}{L_{n}}\left(\frac{1}{n^{2}} \sum_{i, j=1}^{n} \frac{D_{i} D_{j}}{L_{n}}-1+\frac{1}{n^{2}} \sum_{i, j=1}^{n} \exp \left\{-\frac{D_{i} D_{j}}{L_{n}}\right\}\right)=O_{\mathbb{P}}\left(n^{\frac{1}{\gamma}-1}\right) .
$$

We first consider the term inside the brackets in the left hand side of (10).

$$
\begin{aligned}
& \left|\frac{1}{n^{2}} \sum_{i, j=1}^{n} \frac{D_{i} D_{j}}{L_{n}}-1+\frac{1}{n^{2}} \sum_{i, j=1}^{n} \exp \left\{-\frac{D_{i} D_{j}}{L_{n}}\right\}\right| \\
& \leq \frac{1}{n^{2}}\left|\frac{1}{L_{n}}-\frac{1}{\mu n}\right| \sum_{i, j=1}^{n} D_{i} D_{j} \\
& \quad+\frac{1}{n^{2}} \sum_{i, j=1}^{n}\left|\exp \left\{-\frac{D_{i} D_{j}}{L_{n}}\right\}-\exp \left\{-\frac{D_{i} D_{j}}{\mu n}\right\}\right| \\
& \quad+\left|\frac{1}{n^{2}} \sum_{i, j=1}^{n}\left(\frac{D_{i} D_{j}}{\mu n}-1+\exp \left\{-\frac{D_{i} D_{j}}{\mu n}\right\}\right)\right|
\end{aligned}
$$


Since

$$
\frac{1}{n^{2}} \sum_{i, j=1}^{n}\left|\exp \left\{-\frac{D_{i} D_{j}}{L_{n}}\right\}-\exp \left\{-\frac{D_{i} D_{j}}{\mu n}\right\}\right| \leq \frac{1}{n^{2}}\left|\frac{1}{L_{n}}-\frac{1}{\mu n}\right| \sum_{i, j=1}^{n} D_{i} D_{j},
$$

it follows from Corollary 1 that both (11) and (12) are $O_{\mathbb{P}}\left(n^{\frac{1}{\gamma}-2}\right)$. Next, observe that the function $e^{-x}-1+x$ is positive which implies, by Markov's inequality, that (13) scales as its average

$$
\frac{\mathbb{E}\left[D_{1} D_{2}\right]}{\mu n}-1+\mathbb{E}\left[\exp \left\{-\frac{D_{1} D_{2}}{\mu n}\right\}\right] .
$$

where $D_{1}$ and $D_{2}$ are two independent random variables with distribution (10) and $1<\gamma<2$, so that the product $D_{1} D_{2}$ again has distribution (1) with the same exponent, see for instance the Corollary after Theorem 3 in [9]. Now we use Theorem 3 to find that (14), and hence (13) are $O_{\mathbb{P}}\left(n^{-\gamma}\right)$. Finally, the term outside of the brackets in (10) is $O_{\mathbb{P}}(n)$ and since $1-\gamma<\frac{1}{\gamma}-1$ for all $\gamma>1$, the result follows.

\section{Discussion}

The configuration model is one of the most important random graph models developed so far for constructing test graphs, used in the study of structural properties of, and processes on, real-world networks. The model is of course most true to reality when it produces a simple graph. Because this will happen with vanishing probability for most networks, since these have infinite degree variance, the ECM can be seen as the primary model for a neutrally wired simple graph with scale-free degrees. The fact that the fraction of erased edges is vanishing, suffices for obtaining asymptotic structural properties and asymptotic behavior of network processes in the ECM. However, real-world networks are finite, albeit very large. Therefore, it is important to understand and quantify how the properties and processes in a finite network are affected by the fact that the graph is simple.

This paper presents the first step in this direction by providing probabilistic upper bounds for the number of the erased edges in the undirected ECM. This second order analysis shows that the average number of erased edges by the ECM decays as $n^{-1}$ when the variance of the degrees is finite. Since the ECM is computationally less expensive then the RCM and other sequential algorithms, this is a strong argument for using the ECM as a standard model for generating test graphs with given degree distribution. Especially since, in contrast to MarkovChain Monte Carlo methods using edge swap mechanics, it is theoretically well analyzed. We also uncover a new transition in the scaling of the average number of erased edges for regularly varying degree distributions with only finite mean, in terms of the exponent of the degree distribution.

Based on the empirical results found by us in [16, we conjecture that the bounds we obtained are tight, up to some slowly varying functions. Therefore, 
as a next step one could try to prove Central Limit Theorems for the number of erased edges, using the bounds from Theorem 1 as the correct scaling factors. These tools would make it possible to perform statistical analysis of properties on networks, using the ECM as a model for generating test graphs.

\section{References}

[1] Andersson, H.: Limit theorems for a random graph epidemic model. Annals of Applied Probability pp. 1331-1349 (1998)

[2] Artzy-Randrup, Y., Stone, L.: Generating uniformly distributed random networks. Physical Review E 72(5), 056708 (2005)

[3] Bingham, N., Doney, R.: Asymptotic properties of supercritical branching processes i: The galton-watson process. Advances in Applied Probability pp. 711-731 (1974)

[4] Blitzstein, J., Diaconis, P.: A sequential importance sampling algorithm for generating random graphs with prescribed degrees. Internet Mathematics 6(4), 489-522 (2011)

[5] Bollobás, B.: A probabilistic proof of an asymptotic formula for the number of labelled regular graphs. European Journal of Combinatorics 1(4), 311-316 (1980), http://www.sciencedirect.com/science/article/pii/S0195669880800308

[6] Britton, T., Deijfen, M., Martin-Löf, A.: Generating simple random graphs with prescribed degree distribution. Journal of Statistical Physics 124(6), 1377-1397 (2006)

[7] Cooper, C., Dyer, M., Greenhill, C.: Sampling regular graphs and a peer-to-peer network. Combinatorics, Probability and Computing 16(04), 557-593 (2007)

[8] Del Genio, C.I., Kim, H., Toroczkai, Z., Bassler, K.E.: Efficient and exact sampling of simple graphs with given arbitrary degree sequence. PloS one 5(4), e10012 (2010)

[9] Embrechts, P., Goldie, C.M.: On closure and factorization properties of subexponential and related distributions. Journal of the Australian Mathematical Society (Series A) 29(02), 243-256 (1980)

[10] van den Esker, H., van der Hofstad, R., Hooghiemstra, G., Znamenski, D.: Distances in random graphs with infinite mean degrees. Extremes 8(3), 111-141 (2005)

[11] Ferreira, S.C., Castellano, C., Pastor-Satorras, R.: Epidemic thresholds of the susceptible-infected-susceptible model on networks: A comparison of numerical and theoretical results. Physical Review E 86(4), 041125 (2012)

[12] van der Hofstad, R.: Random graphs and complex networks. Unpublished manuscript (2007), http://www.win.tue.nl/ rhof stad/NotesRGCN.pdf

[13] van der Hofstad, R., Hooghiemstra, G., Van Mieghem, P.: Distances in random graphs with finite variance degrees. Random Structures \& Algorithms 27(1), 76 $123(2005)$

[14] van der Hofstad, R., Hooghiemstra, G., Znamenski, D.: Distances in random graphs with finite mean and infinite variance degrees. Eurandom (2005)

[15] van der Hoorn, P., Litvak, N.: Convergence of rank based degree-degree correlations in random directed networks. Moscow Journal of Combinatorics and Number Theory 4(4), 45-83 (2014), http://mjcnt.phystech.edu/en/article.php?id=92

[16] van der Hoorn, P., Litvak, N.: Phase transitions for scaling of structural correlations in directed networks. arXiv preprint arXiv:1504.01535 (2015) 
[17] Lee, H.K., Shim, P.S., Noh, J.D.: Epidemic threshold of the susceptible-infectedsusceptible model on complex networks. Physical Review E 87(6), 062812 (2013)

[18] Maslov, S., Sneppen, K.: Specificity and stability in topology of protein networks. Science 296(5569), 910-913 (2002)

[19] Molloy, M., Reed, B.: A critical point for random graphs with a given degree sequence. Random structures \& algorithms 6(2-3), 161-180 (1995), http://onlinelibrary.wiley.com/doi/10.1002/rsa.3240060204/full

[20] Molloy, M., Reed, B.: The size of the giant component of a random graph with a given degree sequence. Combinatorics, probability and computing 7(03), 295-305 (1998)

[21] Newman, M.E., Strogatz, S.H., Watts, D.J.: Random graphs with arbitrary degree distributions and their applications. Physical Review E 64(2), 026118 (2001), http://journals.aps.org/pre/abstract/10.1103/PhysRevE.64.026118

[22] Schlauch, W.E., Horvát, E.A., Zweig, K.A.: Different flavors of randomness: comparing random graph models with fixed degree sequences. Social Network Analysis and Mining 5(1), 1-14 (2015)

[23] Tabourier, L., Roth, C., Cointet, J.P.: Generating constrained random graphs using multiple edge switches. Journal of Experimental Algorithmics (JEA) 16, 1-7 (2011)

[24] Whitt, W.: Stochastic-process limits: an introduction to stochastic-process limits and their application to queues. Springer (2002) 